\title{
Downregulation of COUP-TFII inhibits glioblastoma growth via targeting MPC1
}

\author{
BING XIAO*, YANGHUA FAN*, MINHUA YE, SHIGANG LV, \\ BIN XU, YI CHAI, MIAOJIN WU and XINGEN ZHU
}

Department of Neurosurgery, Second Affiliated Hospital of Nanchang University, Nanchang, Jiangxi 330006, P.R. China

Received November 7, 2016; Accepted March 14, 2018

DOI: $10.3892 / \mathrm{ol} .2018 .8601$

\begin{abstract}
Chicken ovalbumin upstream promoter transcription factor II (COUP-TFII) is a steroid receptor that is broadly expressed in many tissues throughout embryonic development. Previous studies indicated that COUP-TFII is dysregulated in multiple types of cancer and serves crucial roles in cancer development. The mitochondrial pyruvate carrier 1 (MPC1) is involved in transporting pyruvate for entry into the citric acid cycle, an important event in cancer progression. However, the roles of COUP-TFII and MPC1 in glioma remain unknown. In the present study, it was demonstrated that MPC1 is downregulated in glioblastoma. Furthermore, the inhibition of COUP-TFII was able to increase MPC1 expression and inhibit the growth of glioblastoma cells in vitro and in vivo. The findings from the present study demonstrated that downregulation of COUP-TFII inhibits glioblastoma growth via targeting MPC1. Therefore, COUP-TFII is a potential therapeutic target for glioma.
\end{abstract}

\section{Introduction}

The human brain glioblastoma is one of the most common malignant tumors in the central nervous system, and the median survival time of patients with glioblastoma is typically $<1$ year (1). Although considerable progress has been made in the biological research and treatment of cancer, the study of glioblastoma has not been thoroughly explored, and its exact pathogenesis remains unclear. The main treatment for glioblastoma is surgery, in combination with postoperative

Correspondence to: Dr Miaojin Wu or Professor Xingen Zhu, Department of Neurosurgery, Second Affiliated Hospital of Nanchang University, 1 Minde Road, Nanchang, Jiangxi 330006, P.R. China

E-mail: 905327378@qq.com

E-mail: zxg2008vip@163.com

*Contributed equally

Key words: chicken ovalbumin upstream promoter transcription factor II, mitochondrial pyruvate carrier 1, glioblastoma, proliferation radiation, chemotherapy and immunotherapy. However, the overall treatment effect is poor with a high tumor recurrence rate (2). Therefore, there is an urgent requirement to investigate the underlying molecular mechanisms of glioblastoma progression.

Chicken ovalbumin upstream promoter transcription factor II (COUP-TFII) is a member of the steroid receptor superfamily and is an orphan nuclear receptor (3). COUP-TFII regulates numerous key biological processes, including cell fate determination and organ development (4). COUP-TFII is expressed in multiple tissues and is important for organogenesis (5). There is a growing number of studies that demonstrate that COUP-TFII is dysregulated in multiple cancer types and is crucial for cancer initiation and progression (6-9). However, the role of COUP-TFII in glioma remains unknown.

Pyruvic acid is one of the most important metabolites in eukaryotic cells, which is transported into the mitochondria of differentiated mammalian cells. Pyruvic acid then undergoes oxidative phosphorylation for ATP production. However, cancer cells exhibit an increased dependence on the glycolytic pathway for ATP generation (10), referred to as the Warburg effect (11). Pyruvate metabolism and synthesis have a key role in the Warburg effect,

The mitochondrial pyruvate carrier (MPC), in polymer form, has an important role in mitochondrial pyruvate metabolism $(12,13)$. The MPC gene encodes two proteins-MPC1 and MPC2-and existing research indicates that the MPC gene is downregulated in a variety of tumors, including colon, kidney (14) and prostate cancer (7), and is closely associated with prognosis. MPC serves an important function in the initiation and progression of tumor development; however, its function in glioma remains to be fully elucidated.

In the present study, it was observed that MPC1 was downregulated in glioblastoma and the PROMO TRANSFAC software, predicted that transcriptional binding sites are present in MPC1 for COUP-TFII. Therefore, the aim of the present study was to investigate the COUP-TFII-mediated regulation of $\mathrm{MPC1}$ in glioblastoma. The data indicated that downregulating COUP-TFII expression was able to inhibit glioblastoma growth in vitro and in vivo, which may be mediated by targeting MPC1. These findings support the possibility that COUP-TFII may be a novel therapeutic target for glioblastoma treatment. 


\section{Materials and methods}

Cell culture and reagents. The U87 human glioblastoma cell line and normal brain cell line (HEB) were obtained from the Basic Medical Institute, Chinese Academy of Medical Sciences (Beijing, China). U87 cells were cultured in Dulbecco's modified Eagle's medium (DMEM; Hyclone; GE Healthcare, Chicago, IL, USA) supplemented with $10 \%$ heat-inactivated fetal bovine serum (FBS; Hyclone) at $37^{\circ} \mathrm{C}$ in a humidified atmosphere with $5 \% \mathrm{CO}_{2}$. The $\mathrm{U} 87$ cell line is known to be misidentified/cross-contaminated with an unknown cell line. Despite the reported misidentification/contamination of the U87 cells used in the present study, this cell line is suitable for this current research project, as it is likely a glioblastoma cell line, and therefore exhibits a similar biological nature (15).

Cell transfection. U87 cells were seeded in a 6-well plate at a density of $4.8 \times 10^{5}$ cells/well and cultured for $24 \mathrm{~h}\left(37^{\circ} \mathrm{C}\right)$ until the cells obtained $40 \%$ confluence. The cells were transfected with lentiviruses, with a small interfering-COUP-TFII (100 nM) sequence and scrambled siRNA negative control (siNC, 100 nM), (Shanghai GenePharma Co, Ltd., Shanghai, China) using Lipofectamine ${ }^{\circledR} 2,000$ (Invitrogen; Thermo Fisher Scientific, Inc., Waltham, MA, USA) according to the manufacturer's protocol.

RNA extraction and reverse transcription-quantitative polymerase chain reaction ( $R T-q P C R)$ analysis. Total RNA was extracted from U87 cells or tumors at $48 \mathrm{~h}$ post-transfection using TRIzol (Invitrogen; Thermo Fisher Scientific, Inc.) according to the manufacturer's protocol. RT-qPCR was performed to detect the expression levels of COUP-TFII and MPC1 mRNA. Total RNA was reverse transcribed using a one-step RT-PCR kit (Takara Bio, Inc., Otsu, Japan) according to the manufacturer's protocol.qPCR of COUP-TFII and MPC1 were performed with $5 \mu 1$ COUP-TFII and MPC1 cDNA, using SYBR green (Invitrogen; Thermo Fisher Scientific, Inc.). The reaction conditions were as follows: $93^{\circ} \mathrm{C}$ for $1 \mathrm{~min}, 55^{\circ} \mathrm{C}$ for $1 \mathrm{~min}$, and $72^{\circ} \mathrm{C}$ for $1 \mathrm{~min}$, for 40 cycles. The primer sequences for COUP-TFII and MPC1 were as follows: COUP-TFII forward, 5'-CGGGTGGTCGCCTTTATGG-3'; and reverse 5'-ACAGGCATCTGAGGTGAACAG-3'; MPC1 forward, 5'-ATTTGCCTACAAGGTACAGCC-3'; and reverse 5'-AGT CATCTCGTGTTTGATAAGCC-3', (Shanghai GenePharma Co., Ltd.). The relative expression levels of COUP-TFII and MPC1 were quantified using the $2^{-\Delta \Delta \mathrm{Cq}}$ method (16). Glyceraldehyde 3-phosphate dehydrogenase (GAPDH) served as the internal control.

The primer sequences of GAPDH were as follows: Forward, 5'-GGTGAAGGTCGGAGTCAACG-3', and reverse, 5'-CAAAGTTGTCATGGATGHACC-3'.

Luciferase reporter assay. The U87 cells were cultured at a density of 10,000 cells/well in 96-well plates, and infected with $5 \mu \mathrm{l}$ lenti-si COUP-TFII or lenti-siNC $(6 \mu \mathrm{g} / \mathrm{ml})$, and incubated with $2 \mu$ l Lipofectamine ${ }^{\circledR} 2,000$ and $0.2 \mu \mathrm{g}$ MPC1 luciferase reporter vectors (Thermo Fisher Scientific, Inc.). Following $48 \mathrm{~h}$ of incubation, luciferase activity was measured using a Dual-Luciferase Reporter system (Promega Corporation, Madison, WI, USA). The luciferase enzyme activity was normalized to the Renilla luciferase enzyme activity, All controls used were untreated cells.

Cell proliferation. U87 cells were digested by trypsin (Thermo Fisher Scientific, Inc.) after $48 \mathrm{~h}$ post-transfection and seeded at a density of $5 \times 10^{3}$ cells/well in a 12-well plate. The cells were dived into three groups: Blank control group, si COUP-TFII group and siNC group. The cell cultures were maintained $\left(37^{\circ} \mathrm{C}\right)$ and counted for 6 days under a light microscope (x100 magnification, Olympus Corporation, Tokyo, Japan).

Xenografts. U87 cells $\left(3 \times 10^{6}\right)$, transfected with si COUP-TFII or siNC and mixed with Matrigel were subcutaneously injected into the abdomen of 4-week-old $12 \mathrm{~g}$ male SCID mice (Institute of Zoology, Chinese academy of Sciences, Beijing, China). A total of 20 mice were maintained in a specific pathogen free facility with a constant humidity (40-70\%) and temperature $\left(21 \pm 2^{\circ} \mathrm{C}\right)$, in a $12 / 12 \mathrm{~h}$ light/dark cycle with free access to food and water. The tumor size was measured using a caliper 2 weeks later until the termination of the experiment. All data was recorded, and the formula $\mathrm{v}=0.5 \mathrm{xaxb}^{2}$ (v, tumor volume; a, major diameter of the tumor; $\mathrm{b}$, minor diameter) was used to calculate tumor volume. The mice were sacrificed at the end of the experiment, or if tumor size reached $15 \mathrm{~mm}$ diameter, and tumor tissue was removed from mice for further examination. A portion of the tumor tissue was collected, embedded in paraffin and cut into sections. All animal experiments were approved by the Institutional Animal Care and Use Committee (Nanchang University, Jiangxi, China).

Immunohistochemistry. The tissue sections of 5- $\mu \mathrm{m}$ thickness were routinely deparaffinized. Antigen retrieval was performed in $1 \mathrm{mM}$ EDTA buffer ( $\mathrm{pH} \mathrm{8.0)}$ for $10 \mathrm{~min}$ at $95-100^{\circ} \mathrm{C}$. The sections were rinsed three times in phosphate-buffered saline (PBS) and then blocked in PBS containing 3\% normal horse serum (Thermo Fisher Scientific, Inc.) for $30 \mathrm{~min}$. Subsequently, the sections were incubated with an anti-Ki-67 primary antibody (1:100; catalog no. ab66155; Abcam, Cambridge, UK) overnight at room temperature. The following day, the slides were rinsed in PBS and incubated with a biotinylated goat anti-mouse IgG (dilution, 1:100; catalog no. sc-2039; Santa Cruz Biotechnology, Inc., TX, USA) secondary antibody at $37^{\circ} \mathrm{C}$ for $40 \mathrm{~min}$. The slides were then incubated with ABC-peroxidase reagents (Santa Cruz Biotechnology, Inc.) at $37^{\circ} \mathrm{C}$ for $1 \mathrm{~h}$. Antigen-antibody complexes were detected using a 3,3'-diaminobenzidine horseradish peroxidase color development kit (Beyotime Institute of Biotechnology, Shanghai, China) and evaluated under a light microscope (CX21BIM-SET6; Olympus Corporation, Tokyo, Japan).

Statistical analysis. The data are presented as the mean \pm standard deviation. Data were statistically analyzed by $\chi^{2}$-test and one-way analysis of variance using SPSS software (version 11.5; SPSS Inc., Chicago, IL, USA), The Student-Newman-Keuls post-hoc test was used following the analysis of variance for multiple comparisons. $\mathrm{P}<0.05$ was considered to indicate a statistically significant difference. Each experiment was repeated 3 times. 
A

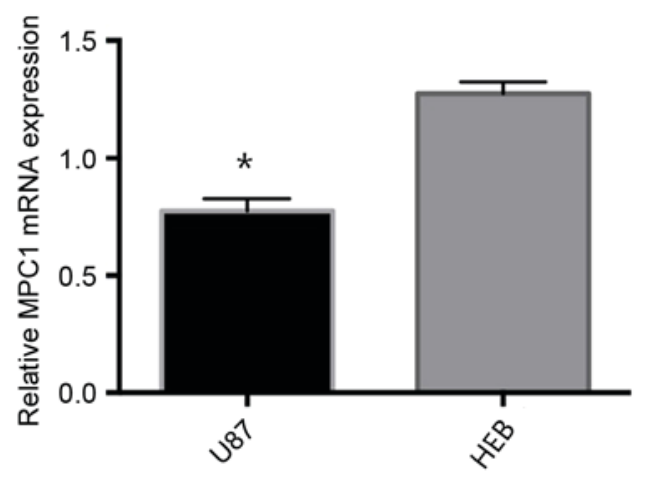

B

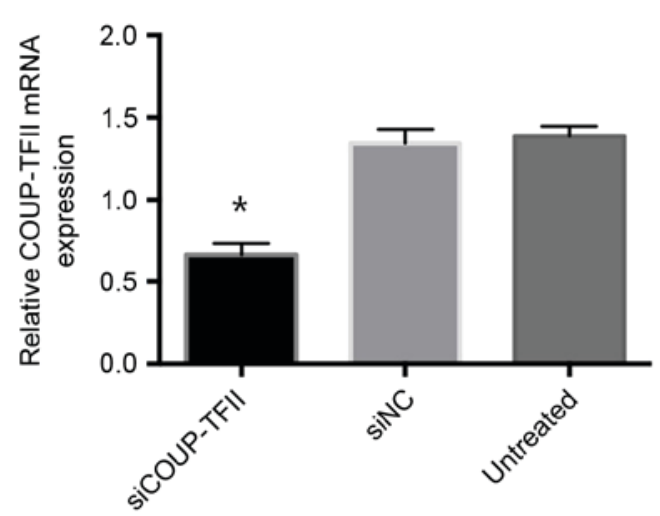

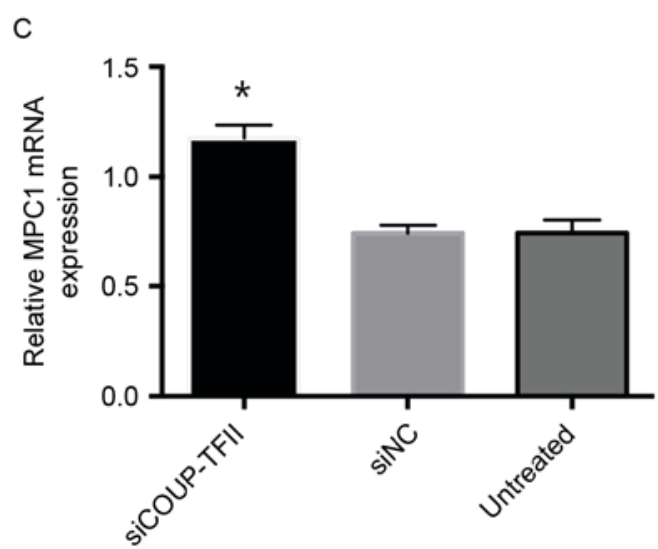

Figure 1. MPC1 is downregulated in U87 cells and upregulated by COUP-TFII suppression. (A) MPC1 mRNA expression was significantly decreased in U87 cells compared with normal brain cell line (HEB). ${ }^{*} \mathrm{P}<0.05$ vs. the normal brain cell line. (B) COUP-TFII mRNA expression was significantly decreased by siCOUP-TFII in U87 cells compared with siNC and untreated cells. "P $<0.05$ vs. siNC and untreated group (C) MPC1 mRNA was significantly increased by siCOUP-TFII in U87 cells compared with siNC and untreated, ${ }^{*} \mathrm{P}<0.05$ vs. siNC and untreated group as evaluated by a reverse transcription-quantitative polymerase chain reaction. COUP-TFII, chicken ovalbumin upstream promoter transcription factor II; MPC1, mitochondrial pyruvate carrier 1; NC, negative control; si, small-interfering.

\section{Results}

MPC1 is downregulated in U87 cells and upregulated when COUP-TFII is suppressed. The expression of MPC1 in U87 cells was examined by RT-qPCR, and MPC1 mRNA expression was significantly decreased in U87 cells compared with normal brain cells (Fig. 1A). Following knockdown of COUP-TFII by siCOUP-TFII in U87 cells, COUP-TFII mRNA expression (Fig. 1B) was significantly decreased in siCOUP-TFII-transfected cells compared with siNC and untreated cells. Also, RT-qPCR analysis revealed that MPC1 mRNA levels were significantly increased in siCOUP-TFII-transfected U87 cells compared with siNC and untreated cells (Fig. 1C). These results indicated that MPC1 was downregulated in U87 cells, and transfection with siCOUP-TFII was able to effectively inhibit COUP-TFII expression. Furthermore, MPC1 mRNA expression was upregulated in response to COUP-TFII suppression.

Downregulation of COUP-TFII suppresses cell proliferation via targeting $M P C 1$. PROMO TRANSFAC predicted that MPC1 has transcriptional binding sites for COUP-TFII (17). To determine whether the prediction was correct, U87 cells were infected with siCOUP-TFII and transfected with MPC1 luciferase reporter vectors. Luciferase activity was measured using a dual-luciferase reporter system. The luciferase activity was significantly reduced in U87 cells that were infected with siCOUP-TFII compared with untreated cells or cells that were infected with siNC (Fig. 2A). This result indicated that MPC1 might be a direct target of COUP-TFII. Furthermore, the effect of COUP-TFII on cell proliferation was investigated, and it was demonstrated that U87 cell proliferation was inhibited by siCOUP-TFII compared with siNC and the untreated group (Fig. 2B). Therefore, these findings indicated that the downregulation of COUP-TFII suppresses U87 cell proliferation via targeting MPC.

SiCOUP-TFII works efficiently in an in vivo animal model. To test the efficiency of COUP-TFII knockdown in a mouse model, siCOUP-TFII and si-NC-transfected U87 cells were injected into the abdomen of SCID mice. The efficiency of siCOUP-TFII was good. COUP-TFII mRNA expression was significantly downregulated by siCOUP-TFII compared with the control group as evaluated by RT-qPCR (Fig. 3A). However, MPC1 mRNA expression was significantly increased by siCOUP-TFII compared with the control group (Fig. 3B). These findings indicated that the injection of 

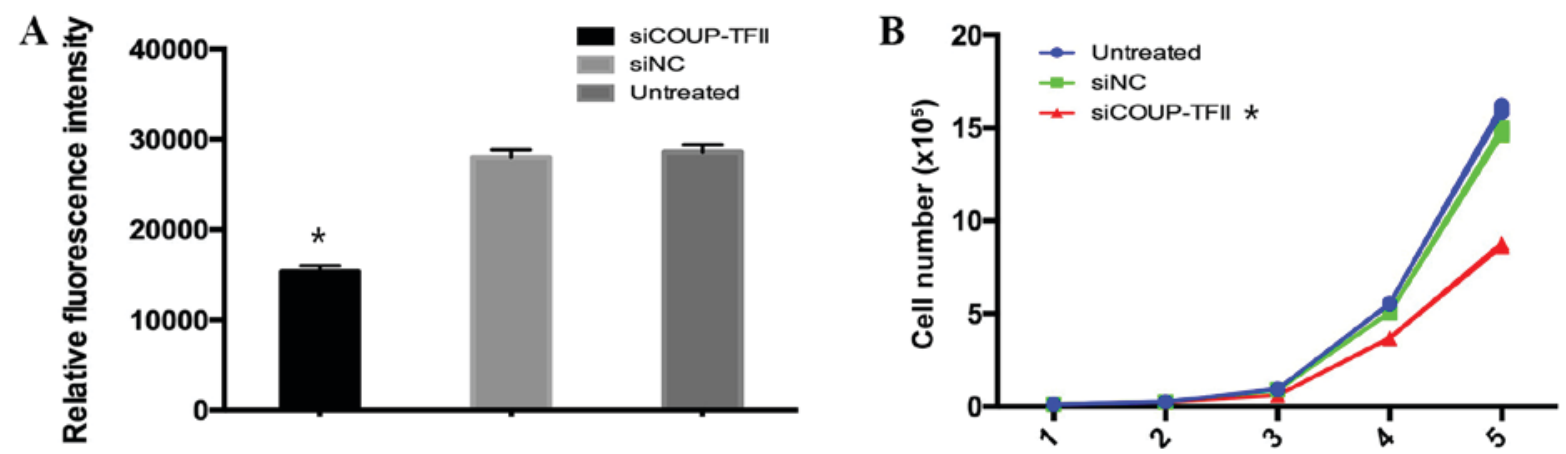

Figure 2. Downregulation of COUP-TFII suppresses cell proliferation via targeting MPC1. (A) Luciferase activity was significantly reduced in U87 cells that were infected with siCOUP-TFII compared with untreated cells or cells that were infected with siNC. "P<0.05 vs. untreated cells or siNC group. (B) U87 cell proliferation is inhibited by siCOUP-TFII compared with siNC or the untreated group as demonstrated by cell counting, $\mathrm{P}<0.01$ vs. siNC or the untreated group. COUP-TFII, chicken ovalbumin upstream promoter transcription factor II; MPC1, mitochondrial pyruvate carrier 1; NC, negative control; si, small-interfering.

A

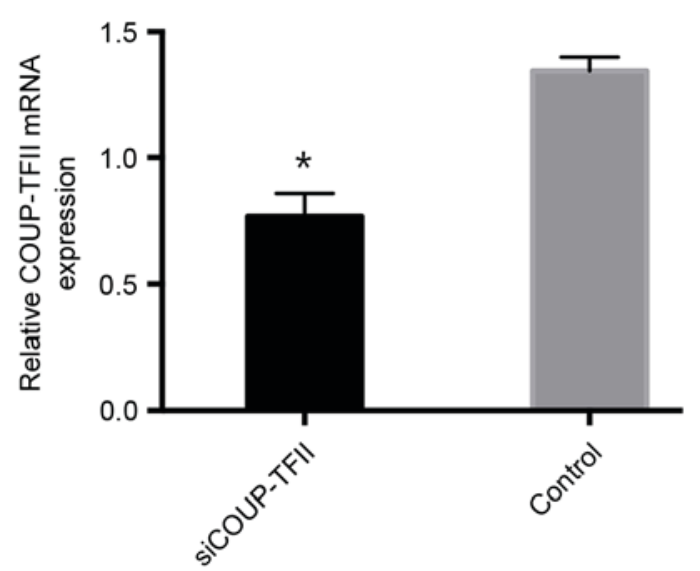

B

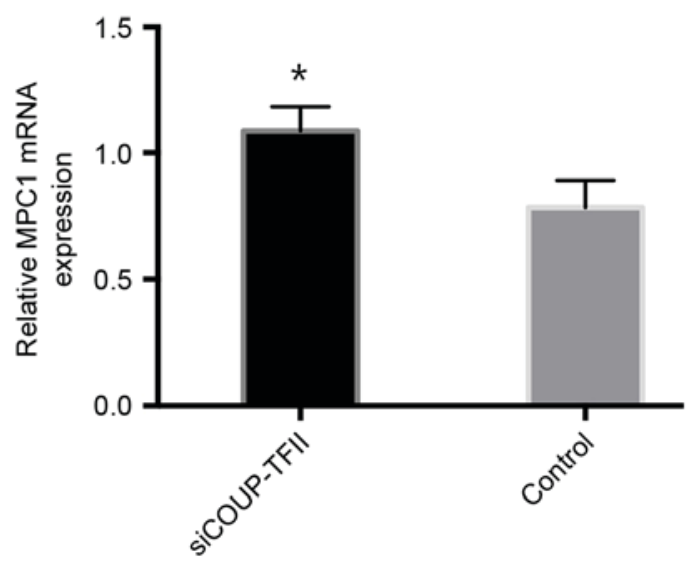

Figure 3. siCOUP-TFII works efficiently in an in vivo animal model. (A) The expression of COUP-TFII was significantly downregulated by siCOUP-TFII compared with the control group as evaluated by RT-qPCR. " $\mathrm{P}<0.05$ vs. control. (B) MPC1 mRNA expression was significantly increased by siCOUP-TFII compared with the control group as evaluated by RT-qPCR. " $\mathrm{P}<0.05$ vs. the control. COUP-TFII, chicken ovalbumin upstream promoter transcription factor II; MPC1, mitochondrial pyruvate carrier 1; RT-qPCR, reverse transcription-quantitative polymerase chain reaction; si, small-interfering.

siCOUP-TFII-transfected U87 cells was able to downregulate COUP-TFII expression and upregulate MPC1 in vivo.

Downregulation of COUP-TFII suppresses tumor growth in vivo. To assess the effect of siCOUP-TFII knockdown on tumor volume, xenograft assays in SCID mice were performed. In U87 cell lines, siCOUP-TFII was able to slow down tumor growth throughout the experiment. The size (Fig. 4A), volume (Fig. 4B) and weight (Fig. 4C) of the tumors in the siCOUP-TFII group were markedly smaller compared with the control group. Tumor cell proliferation was then assessed by immunohistochemical detection of $\mathrm{Ki}-67$, and the results were consistent with the in vitro findings. Ki-67 expression was more evident in the control group (Fig. 4D), which indicated that the proliferation of U87 cells in xenograft was inhibited by siCOUP-TFII. These results indicated that the downregulation of COUP-TFII was able to suppress tumor growth in vivo.

\section{Discussion}

In previous years, multiple studies have been conducted concerning COUP-TFII, which has been identified to contribute an essential role in the initiation and progression of cancer. Therefore, COUP-TF11 has been indicated to be a potential therapeutic target for cancer treatment. Wang et al (14) reported that COUP-TFII was upregulated in patients with prostate cancer, which can facilitate a metabolic switch to enhance glycolysis and promote prostate cancer progression. COUP-TFII cooperates with phosphatase and tensin homolog deletion to promote prostate metastasis and progression (15). This observation provides a possibility of targeting COUP-TFII to regulate cancer cell metabolism for prostate cancer therapy.

A growing number of studies support an important role for COUP-TFII in cell fate determination $(4,18,19)$; however, the underlying mechanism, by which COUP-TFII regulates these processes, remains unclear. During differentiation, previous studies have demonstrated that COUP-TFII is regulated by microRNA (miR)-302a, miR-194, sonic hedgehog and Wnt family member 3A (20-23). Lee et al (18) observed that fibroblast growth factor 2 is an inducer of COUP-TFII expression and may suppress the osteogenic potential of mesenchymal cells by inducing COUP-TFII expression. However, the role of COUP-TFII in glioma remains unknown. 
A
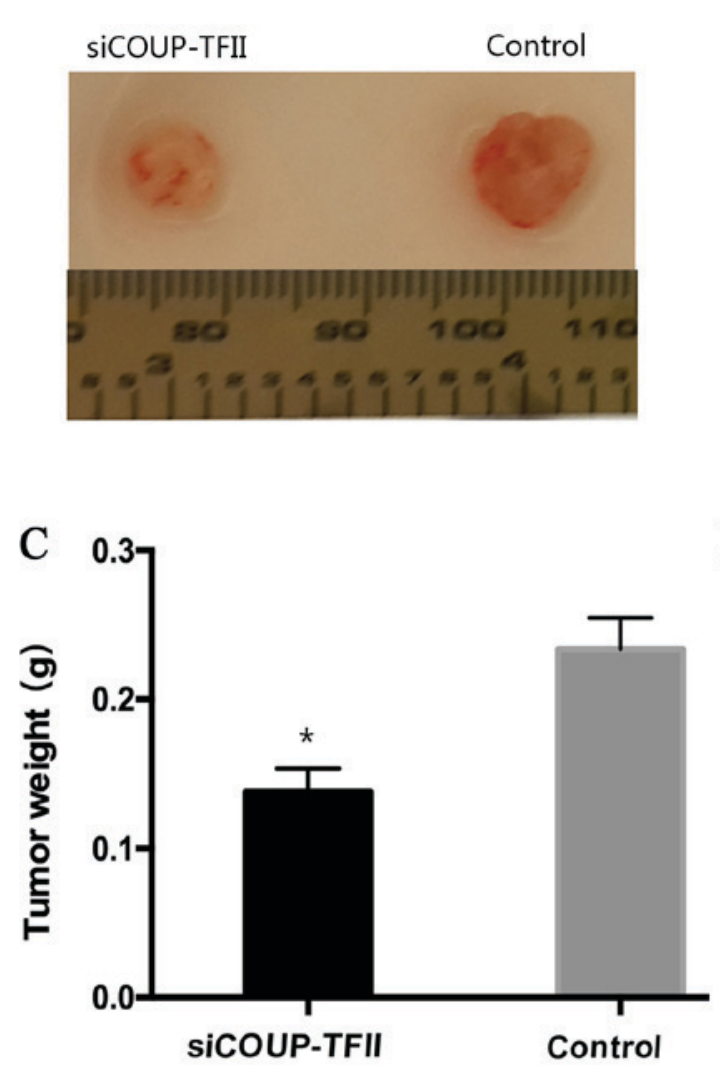

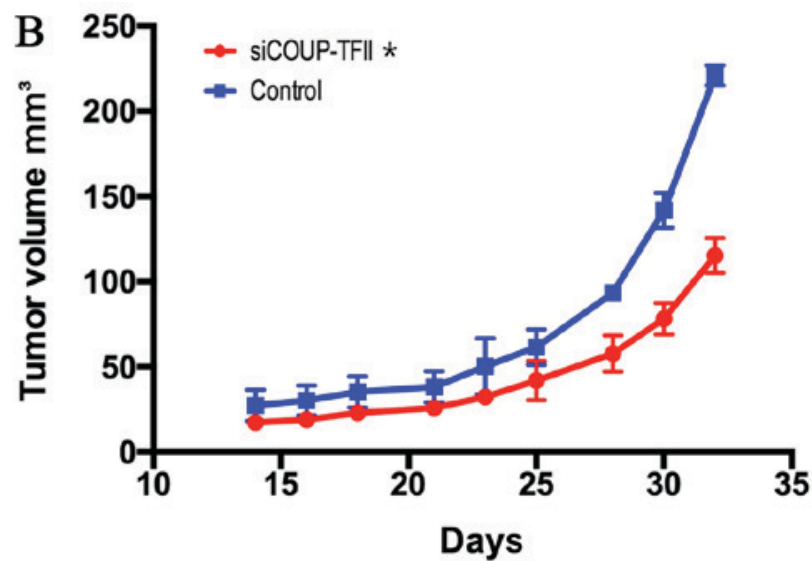

D

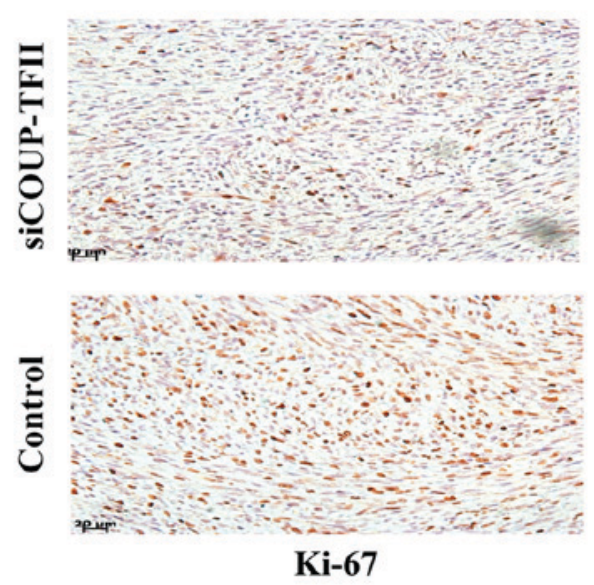

Figure 4. Downregulation of COUP-TFII suppresses tumor growth in vivo. (A) Images of tumors (mm). The (B) tumor volume and (C) weight were significantly decreased in the siCOUP-TFII group compared with the control group ( $\mathrm{P}<0.01)$. (D) Tumor cell proliferation was assessed by immunohistochemical detection of Ki-67. Ki-67 expression was increased in the control group compared with the siCOUP-TFII group. Magnification, x200. COUP-TFII, chicken ovalbumin upstream promoter transcription factor II; si, small-interfering.

COUP-TFII modulates a large number of target genes in various cell types (24), including several genes in the glycolysis pathway. In the present study, bioinformatics analysis predicted that the MPC1 promoter contains the binding site of COUP-TFII, which indicated that MPC1 is regulated by COUP-TFII, and MPC1 was downregulated in U87 glioblastoma cells.

Previously, MPC1 and MPC2 were known as BRP44L and BRP44. It was later reported that MPC1 and MPC2 form a complex that transports pyruvate into mitochondria for further ATP production $(7,12,25)$. Previous studies have indicated that MPC gene was downregulated in a variety of tumors, including colon, kidney (13) and prostate cancer (14), and was closely associated with prognosis. MPC has an important role in the initiation and progression of tumor development, over-expression of MPC was able to significantly inhibit colon cancer xenograft growth, colony formation in soft agar and spheroid formation, indicating that MPC may become a novel tumor therapy target (26). In the present study, in response to COUP-TFII inhibition, MPC1 mRNA expression was increased, and the proliferation of U87 cells was suppressed, The xenograft assay and Ki-67 immunohistochemistry also demonstrated that, in response to COUP-TFII inhibition, tumor growth was inhibited in vivo.

In conclusion, the present study demonstrated that MPC1 was downregulated in glioblastoma and regulated by
COUP-TFII. The inhibition of COUP-TFII may suppress the proliferation of glioblastoma in vitro and in vivo; therefore, COUP-TFII may be a novel therapeutic target of glioblastoma. However, the mechanism underlying the effects of COUP-TFII in glioblastoma remains to be elucidated and more in-depth research is required.

\section{Acknowledgements}

The authors wish to thank the Central laboratory of Second Affiliated Hospital of Nanchang University for lab support.

\section{Funding}

The present study was supported by the National Natural Science Foundation of China (grant no. 81660420), the Construction Plan of the Superior Science and Technology Innovation Team of Jiangxi Province (grant no. 20152BCB24009), and the Foreign Science and Technology Cooperation Plan of Jiangxi Province (grant no. 20151BDH80009).

\section{Availability of data and materials}

All data generated or analyzed during this study are included in this published article. 


\section{Authors' contributions}

XZ and MW conceived and designed the study. BXi, YF, MY, $\mathrm{SL}, \mathrm{BXu}$ and $\mathrm{YC}$ performed the experiments. BXi and YF wrote the paper, MW reviewed and edited the manuscript. All authors read and approved the manuscript.

\section{Ethics approval and consent to participate}

All animal experiments were approved by the Institutional Animal Care and Use Committee (Nanchang University, Jiangxi, China).

\section{Consent for publication}

Not applicable.

\section{Competing interests}

The authors declare that they have no competing interests.

\section{References}

1. Krex D, Klink B, Hartmann C, von Deimling A, Pietsch T, Simon M, Sabel M, Steinbach JP, Heese O, Reifenberger G, et al: Long-term survival with glioblastoma multiforme. Brain 130 : 2596-2606, 2007.

2. Xiao B, Zhou X, Ye M, Lv S, Wu M, Liao C, Han L, Kang C and Zhu X: MicroRNA-566 modulates vascular endothelial growth factor by targeting Von Hippel-Landau in human glioblastoma in vitro and in vivo. Mol Med Rep 13: 379-385, 2016.

3. Lin FJ, Qin J, Tang K, Tsai SY and Tsai MJ: Coup d'Etat: An orphan takes control. Endocr Rev 32: 404-421, 2011.

4. Mi NL, Kim JW, Oh SH, Jeong BC, Hwang YC and Koh JT: FGF2 stimulates COUP-TFII expression via the MEK1/2 pathway to inhibit osteoblast differentiation in C3H10T1/2 Cells. PLoS One 11: e0159234, 2016.

5. Pereira FA, Tsai MJ and Tsai SY: COUP-TF orphan nuclear receptors in development and differentiation. Cell Mol Life Sci 57: 1388-1398, 2000.

6. Eboli ML, Paradies G, Galeotti T and Papa S: Pyruvate transport in tumour-cell mitochondria. Biochim Biophys Acta 460: 183-187, 1977.

7. Bricker DK, Taylor EB, Schell JC, Orsak T, Boutron A, Chen YC, Cox JE, Cardon CM, Van Vranken JG, Dephoure N, et al: A mitochondrial pyruvate carrier required for pyruvate uptake in yeast, Drosophila, and humans. Science 337: 96-100, 2012.

8. Herzig S, Raemy E, Montessuit S, Veuthey JL, Zamboni N, Westermann B, Kunji ER and Martinou JC: Identi cation and functional expression of the mitochondrial pyruvate carrier. Science 337: 93-96, 2012.

9. Yang C, Ko B, Hensley CT, Jiang L, Wasti AT, Kim J, Sudderth J, Calvaruso MA, Lumata L, Mitsche M, et al: Glutamine oxidation maintains the TCA cycle and cell survival during impaired mitochondrial pyruvate transport. Mol Cell 56: 414-424, 2014.

10. Vander Heiden MG, Cantley LC and Thompson CB: Understanding the Warburg effect: The metabolic requirements of cell proliferation. Science 324: 1029-1033, 2009.
11. Warburg O, Wind F and Negelein E: The metabolism of tumors in the body. J Gen Physiol 8: 519-530, 1927.

12. Herzig S, Raemy E, Montessuit S, Veuthey JL, Zamboni N, Westermann B, Kunji ER and Martinou JC: Identification and functional expression of the mitochondrial pyruvate carrier. Science 337: 93-96, 2012.

13. Schell J, Olson K, Jiang L, Hawkins AJ, Van Vranken JG, Xie J, Egnatchik RA, Earl EG, DeBerardinis RJ and Rutter J: A Role for the mitochondrial pyruvate carrier as a repressor of the warburg effect and colon cancer cell growth. Mol Cell 56: 400-413, 2014.

14. Wang L, Xu M, Qin J, Lin SC, Lee HJ, Tsai SY and Tsai MJ: MPC1, a key gene in cancer metabolism, is regulated by COUPTFII in human prostate cancer. Oncotarget 7: 14673-14683, 2016.

15. Qin J, Wu SP, Creighton CJ, Dai F, Xie X, Cheng CM, Frolov A, Ayala G, Lin X, Feng XH, Ittmann MM, et al: COUP-TFII inhibits TGF-beta- induced growth barrier to promote prostate tumorigenesis. Nature 493: 236-240, 2013.

16. Livak KJ and Schmittgen TD: Analysis of relative gene expression data using real-time quantitative PCR and the 2(-Delta Delta C(T)) method. Methods 25: 402-408, 2001.

17. Allen M, Bjerke M, Edlund H, Nelander S and Westermark B: Origin of the U87MG glioma cell line: Good news and bad news. Sci Transl Med 8: 354re3, 2016.

18. Lee S, Kang J, Yoo J, Ganesan SK, Cook SC, Aguilar B, Ramu S, Lee J and Hong YK: Prox1 physically and functionally interacts with COUP-TFII to specify lymphatic endothelial cell fate. Blood 113: 1856-1859, 2009.

19. Wu SP, Yu CT, Tsai SY and Tsai MJ: Choose your destiny: Make a cell fate decision with COUP-TFII. J Steroid Biochem Mol Biol 157: 7-12, 2016.

20. Xu Z, Yu S, Hsu CH, Eguchi J and Rosen ED: The orphan nuclear receptor chicken ovalbumin upstream promoter-transcription factor II is a critical regulator of adipogenesis. Proc Natl Acad Sci USA 105: 2421-2426, 2008.

21. Okamura M, Kudo H, Wakabayashi KI, Tanaka T, Nonaka A, Uchida A, Tsutsumi S, Sakakibara I, Naito M, Osborne TF, et al: COUP-TFII acts down- stream of Wnt/beta-catenin signal to silence PPARgamma gene expression and repress adipogenesis. Proc Natl Acad Sci USA 106: 5819-5824, 2009.

22. Jeong BC, Kang IH, Hwang YC, Kim SH and Koh JT: MicroRNA-194 reciprocally stimulates osteogenesis and inhibits adipogenesis via regulating COUP-TFII expression. Cell Death Dis 5: e1532, 2014.

23. Kang IH, Jeong BC, Hur SW, Choi H, Choi SH, Ryu JH, Hwang YC and Koh JT: MicroRNA-302a stimulates osteo-blastic differentiation by repressing COUP-TFII Expression. J Cell Physiol 230: 911-921, 2015.

24. Tsai SY and Tsai MJ: Chick ovalbumin upstream pro- moter-transcription factors (COUP-TFs): Coming of age. Endocr Rev 18: 229-240, 1997.

25. Trachtulec Z and Forejt J: Synteny of orthologous genes conserved in mammals, snake, fly, nematode, and ssion yeast. Mamm Genome 12: 227-231, 2001.

26. Vacanti NM, Divakaruni AS, Green CR, Parker SJ, Henry RR, Ciaraldi TP, Murphy AN and Metallo CM: Regulation of substrate utilization by the mitochondrial pyruvate carrier. Mol Cell 56: 425, 2014.

c) (†) $\Theta$ This work is licensed under a Creative Commons

Attribution-NonCommercial-NoDerivatives 4.0 International (CC BY-NC-ND 4.0) License. 